\title{
Effective coarse-grained simulations of super-thick multi-walled carbon nanotubes under torsion
}

\author{
Jian Zou, ${ }^{1}$ Xu Huang, ${ }^{1}$ Marino Arroyo, ${ }^{2}$ and Sulin Zhang $^{1, a)}$ \\ ${ }^{1}$ Department of Engineering Science and Mechanics, The Pennsylvania State University, University Park, \\ Pennsylvania 16802, USA \\ ${ }^{2}$ Department of Applied Mathematics 3, LaCàN, Universitat Politècnica de Catalunya (UPC), Barcelona \\ 08034, Spain
}

(Received 6 November 2008; accepted 15 December 2008; published online 10 February 2009)

Under torsion and beyond the buckling point, multi-walled carbon nanotubes (MWCNTs) develop a periodic wave-like rippling morphology. Here, we show that torsional rippling deformations can be accurately described by a simple sinusoidal shape function. Combining this observation with the geometry optimization, we develop an effective coarse-grained model that reproduces the complex nonlinear mechanical responses of thick MWCNTs under torsion predicted by large-scale atomistic simulations. Furthermore, the model allows us to simulate super-thick tubes, inaccessible by other coarse-grained methods. With this effective coarse-grained model, we show from an energetic analysis that the rippling deformation is a result of in-plane strain energy relaxation, penalized by the increase in the interlayer van der Waals interaction energy. Our simulations reveal that the torsional response of MWCNTs with up to 100 layers approximately follows a simple bilinear law, and the ratio of the torsional rigidities in the pre- and post-buckling regimes is nearly a constant, independent of the tube radius. In contrast, the bifurcation torsional strain powerly scales with the tube radius. We also find that the wave number in the circumferential direction linearly increases with tube radius, while the wavelength monotonically increases with tube radius, and approaches a constant in the limit of bulk graphite. The bilinear constitutive relation, together with the scaling law of the bifurcation torsional strain, furnishes a simple nonlinear beam theory, which facilitates the analysis of MWCNT bundles and networks. (C) 2009 American Institute of Physics.

[DOI: $10.1063 / 1.3074285]$

\section{INTRODUCTION}

Owing to their remarkable physical and chemical properties, carbon nanotubes (CNTs) continue to stimulate experimental $^{1}$ and theoretical studies. ${ }^{2}$ To date, material properties of single-walled CNTs (SWCNTs), ranging from the elastic constants, the brittle and ductile failure mechanisms, to the nucleation mechanisms and the weakening effects of various defects, have been well characterized both theoretically ${ }^{3-17}$ and experimentally. ${ }^{18,19}$ Numerical methods adopted in these analyses span all the length scales including quantum mechanical calculations, ${ }^{3}$ atomistic simulations with empirical hydrocarbon potentials, ${ }^{4}$ and simple thin shell and beam theories. ${ }^{5}$ While short SWCNTs are accessible to atomistic simulations with empirical hydrocarbon potentials or even quantum mechanical calculations, numerical studies on multi-walled CNTs (MWCNTs) with atomic resolutions remain challenging, largely due to the prohibitive computational cost in computing the interlayer van der Waals interactions. ${ }^{14-17}$ Typical atomistic simulations are limited to a system consisting of only a few layers with a tube length on the order of $10 \mathrm{~nm}$, and thus incapable of probing longrange elastic deformation morphologies of MWCNTs. Linear elastic beam theories have been invoked to extract the mechanical properties from the experimental data and to ana-

\footnotetext{
a) Author to whom correspondence should be addressed. Electronic mail: suz10@psu.edu.
}

lyze CNT-based devices. However, treating CNTs by linear elastic beams often leads to very poor predictions due to their intrinsically nonlinear characteristics. The apparent limitations of atomistic simulations and linear elasticity theories have stimulated continual search for efficient yet accurate methods for the analysis of MWCNTs.

Experimental studies found that MWCNTs are very rigid in their axial direction but very flexible in the transverse direction. These systems can sustain large deformations before bond breaking occurs. ${ }^{18,19}$ These nonlinear elastic deformations suggest that finite crystal elasticity (FCE) theories may be well suited to simulate the deformation of MWCNTs. Indeed, within the framework of FCE, a recently developed method coarse-grains the fully atomistic model by finiteelement discretization of CNTs, where the constitutive relation on the continuum level is seamlessly cast from the interatomic interaction potentials. ${ }^{12-16}$ Different from several other continuum methods, ${ }^{11,20}$ this method overcomes the drawbacks of the standard Cauchy-Born rule in treating curved atomic monolayers and analytically casts the interatomic potentials into the strain energy density function through an exponential mapping. This method (hereafter referred to as FCE) typically reduces the number of degrees of freedom by one to two orders of magnitude, while remaining faithful to the corresponding atomistic model for defect-free CNTs, as demonstrated by a number of examples. ${ }^{12-16}$ Figure 1 demonstrates the accuracy of the FCE calculations by comparing with the fully atomistic simulations of the deforma- 
(a)

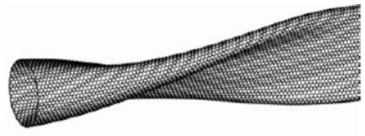

(b)

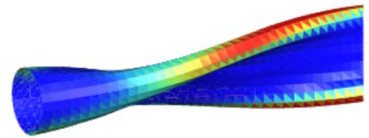

$50^{\circ}$
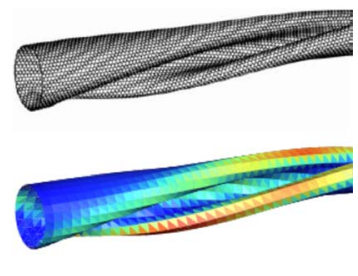

$75^{\circ}$

FIG. 1. (Color online) Twisted $37.67 \mathrm{~nm}$ long (30,30) nanotube: comparison between the atomistic model and the FCE model for two twisting angles. The atomistic system has 54000 degrees of freedom while the continuum model has only 5070. The computational time with the continuum approach is seven times smaller than the full atomistic simulations, while the strain energy predicted by FCE calculations is only $\sim 2 \%$ different from full atomistic simulations at $75^{\circ}$ twisting and can be lower with a refined mesh. (a) Superimposed deformation configurations for atomistic (black spheres) and FCE (gray surface) calculations and (b) map of the strain energy density on the finite element computational mesh (red is high, blue is low).

tion morphologies of a twisted $(30,30)$ SWCNT at two twisting angles of $50^{\circ}$ and $75^{\circ}$. However, the method is still computationally very intensive when simulating very thick MWCNTs. ${ }^{21}$

Recent numerical studies ${ }^{14-16,22}$ using the FCE method have found that under torsion or bending MWCNTs exhibit well defined rippling deformation patterns beyond the buckling point. These distributed patterns are associated with a nonlinear elastic (recoverable) behavior and distinctly different from the localized kinks for twisted or bent SWCNTs. ${ }^{12}$ The periodicity and regularity of the rippling pattern along both the tube length and circumferential directions invite even coarser description of MWCNTs with little compromise of simulation accuracy. In this paper, we characterize the rippling morphology of MWCNTs under torsion using a simple sinusoidal shape function with only several yet-todetermine parameters that measure the rippling amplitudes of each layer and the wave numbers along the axial and circumferential directions. An energy minimization scheme ${ }^{23}$ is then utilized to determine these parameters from which the energetics and the evolution of the deformation can be extracted. We show that this coarse-grained model is computationally very effective and able to reproduce the rippling deformation morphology and the mechanical properties of twisted MWCNTs predicted by FCE simulations. Our analysis with this effective coarse-grained method shows that the circumferential wave number increases nearly linearly with respect to the tube radius while the bifurcation torsional strain powerly scales with tube radius. Although each layer deforms highly nonlinearly, the collective mechanical response of MWCNTs during the entire torsional process approximately follows a simple bilinear law. We find that the ratio of the torsional rigidities between the pre- and post-bulking regimes is nearly a constant, independent of the tube size. The bilinear constitutive relation, together with the scaling law of the bifurcation torsional strain, constitutes a simple nonlinear beam theory, which facilitates large-scale simulations of MWCNT bundles and networks. ${ }^{24-26}$

The rest of the paper is organized as follows. We first show that the rippling morphology of a twisted MWCNT can be well characterized by a sinusoidal shape function in the

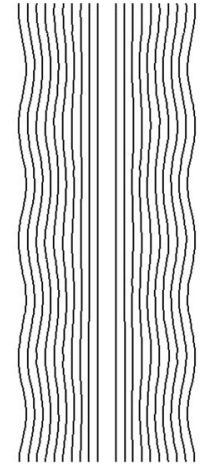

(a)

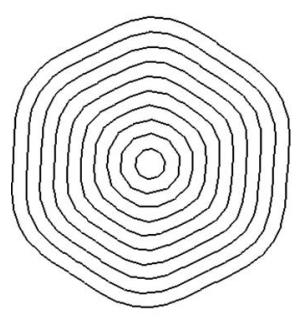

(c)

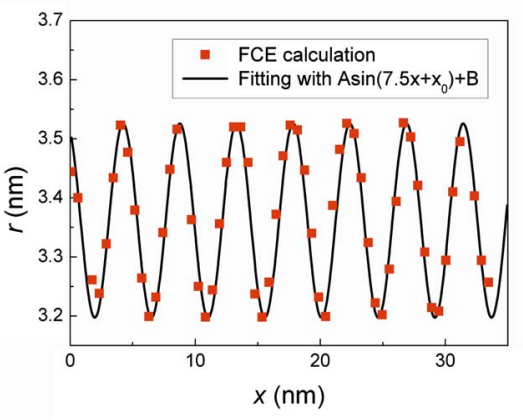

(b)

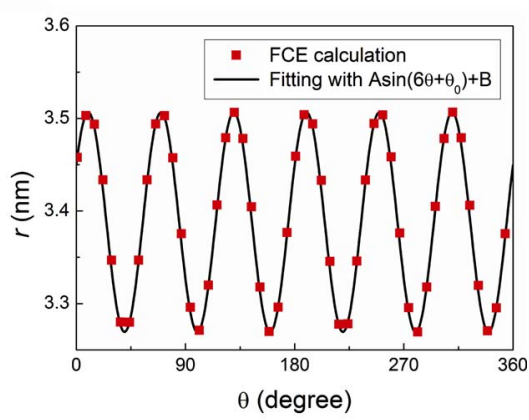

(d)
FIG. 2. (Color online) Rippling morphologies of a ten-walled MWCNT under torsion obtained by FCE simulations (Ref. 22) can be approximated by a simple sinusoidal function. (a) Longitudinal-section view of the deformation morphology. (b) Radial coordinates $r$ of the sample points (red symbols) in the outermost layer $(50,50)$ as a function of axial coordinates $x$ fitted by a sinusoidal function (black curve). (c) Cross-sectional view. (d) Radial coordinates $r$ of the sample points (red symbols) as a function of the polar angle $\theta$ fitted by a sinusoidal function (black curve).

post-buckling regime. Based on the sinusoidal shape function and the energy minimization scheme, the coarse-grained model is furnished and validated by comparing with the FCE method. The deformation morphologies and mechanical responses of MWCNTs with up to 100 layers are then systematically documented. Finally, we conclude our findings and comment on the coarse-grained method.

\section{METHODOLOGY}

Since the accuracy of the energy minimization scheme depends on that of the shape function in approximating the deformation morphology, we begin with examining the rippling morphology of a ten-walled MWCNT under torsion using the FCE method. ${ }^{22}$ From hereafter, an $n$-walled MWCNT is indexed by $(5,5) @(10,10) @ \ldots @(5 n, 5 n)$, with $n$ being the number of layers; the interlayer spacing is roughly $0.34 \mathrm{~nm}$. It should be noted that with a reasonably fine mesh size, the FCE method can faithfully reproduce the deformation energetics and morphology of MWCNTs of the fully atomistic simulations in the entire deformation regime. ${ }^{12}$ The FCE model consists of about $18 \times 10^{3}$ finite element nodes; the corresponding atomic counterpart has more than $150 \times 10^{3}$ atoms. When the torsional deformation exceeds the bifurcation point, the initially circular cross section of the MWCNT buckles into a hexagonal shape with rounded corners, and the rippling amplitude increases from 
the innermost layer (nearly zero) to the outermost layer, as shown in Figs. 2(a) and 2(c). We sample representative points from the rippled outermost layer and plot the radial coordinate $r$ of these sample points as a function of the axial coordinate $x$ and polar angle $\theta$, respectively. We find that the spatial arrangement of these sample points can be well fitted by a single sinusoidal shape function in both the axial and circumferential directions with a circumferential wave number equal to 6 and an axial wave number of about 7.5 [Figs. 2(b) and 2(d)].

Motivated by the observation and noting that the rippling morphology is helical in nature and nearly uniformly distributed along the MWCNTs, we approximate the rippling morphology of an $n$-walled CNT by the following shape function, as

$$
\begin{aligned}
& x=X, \\
& r=R(i)+B_{0}(i)+B_{1}(i) \sin \left(N_{x} \frac{2 \pi x}{L}-N_{y} \theta\right), \\
& \theta=\Theta+x \phi,
\end{aligned}
$$

where $i=1, \ldots, n$ is the layer index (from innermost to outermost), $L$ is the tube length, and $\phi$ is the torsional angle per unit length; $X, R(i)$, and $\Theta$, respectively, are the reference coordinates along the axial, radial, and polar directions in the undeformed configuration, while $x, r$, and $\theta$ are the corresponding coordinates in the deformed configuration. One notices from Figs. 2(a) and 2(c) that all the layers deform compatibly, which suggests that all the layers share the same wave numbers in both axial and circumferential directions. Accordingly, $N_{x}$ and $N_{y}$ are two global parameters denoting the wave numbers along axial and circumferential directions, respectively, for all the layers; $B_{0}(i)$ and $B_{1}(i)$ are local parameters representing uniform radius change (expansion or shrinkage) and the rippling amplitude, respectively, for the $i$ th layer. The radial expansion (or shrinkage) $B_{0}(i)$ is necessary to break the energetic symmetry of the ridges and furrows in the deformation morphology. ${ }^{22}$ The wave number $N_{y}$ is enforced to be an integer in order to maintain the continuity condition of the deformation morphology along the circumferential direction when the polar angle approaches $2 \pi$. For an $n$-walled CNT, regardless of its length, only $2(n+1)$ independent variables (unknowns) are needed to characterize its deformation morphology, which is much fewer than the number of degrees of freedom in both the FCE and fully atomistic models.

The deformation shape function defined in Eq. (1) facilitates the calculation of the total system energy of the twisted MWCNTs at specified torsional deformation $\phi$. With trial values of the parameters in the deformation shape function, the atomic positions in the deformed configuration are uniquely determined, which yield directly the bond lengths and bond angles in the deformed configuration. In the energy calculation, we adopt the second-generation Brenner potential $^{27}$ to describe the in-plane covalent bonding energy and the classical Lennard-Jones potential ${ }^{28}$ to the interlayer nonbonding van der Waals interaction energy. The constitutive level relaxation of inner displacement ${ }^{12}$ is not imple-

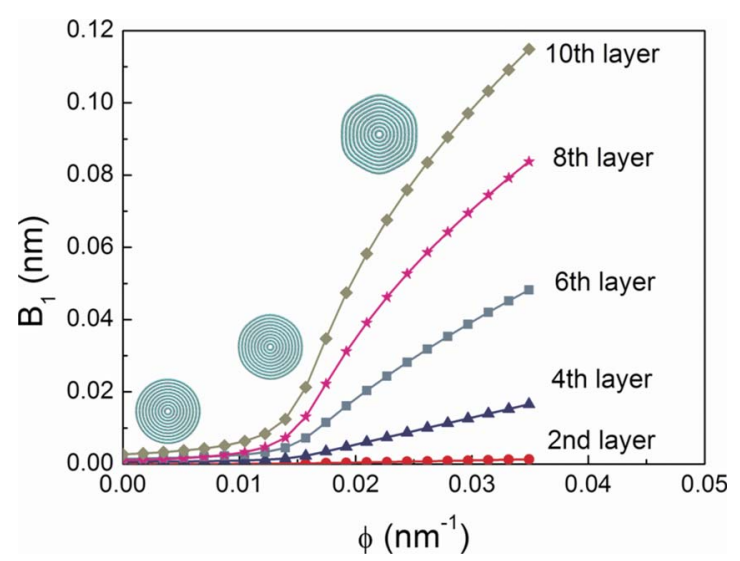

FIG. 3. (Color online) Evolution of rippling amplitude $B_{1}(i)$ for the $i$ th layer $(i=1$ for the innermost and $i=10$ for the outermost) in a ten-walled MWCNT as a function of torsional deformation. The innermost two layers always have vanishing $B_{1}$ acting as the hard core in the entire torsional process due to the strong confinement by the outer layers. The insets depict the evolution of the cross sections from the initial circular shape to an oval and eventually to a hexagon with rounded corners.

mented here. In the simulations, the MWCNT is rotated incrementally by spatially repositioning the tube surfaces uniformly in terms of the rotation angle $\phi$. The equilibrium configuration under specific torsional deformation is obtained by minimizing the total potential energy with respect to $N_{x}, N_{y}, B_{0}(i)$, and $B_{1}(i)(i=1, \ldots, n)$ through the limitedmemory Broyden-Fletcher-Goldfarb-Shanno (BFGS) algorithm. $^{29}$ To begin with, the MWCNT is relaxed at $\phi=0$ with small random nonzero values of $B_{1}(i)$. To ensure fast convergence, the parameters determined from the previous step are used as initial values for the next minimization step. The torsion of bifurcation corresponds to the critical point at which the rippling amplitude $B_{1}$ increases abruptly. The wave number $N_{y}$ is determined by a two-step minimization process. The total system energy is first minimized without constraining $N_{y}$ to an integer. The wave number is then chosen to be the integer close to the value obtained, and the system is again relaxed at the fixed wave number $N_{y}$, which identifies the true energy equilibrium configuration and the values of other parameters. If the value of $N_{y}$ obtained from the first minimization step is equally close to two integers, then the value of $N_{y}$ is chosen to be the one that gives rise to a lower system energy.

\section{SIMULATION RESULTS}

\section{A. Model validation: A representative study}

We next validate the coarse-grain model by a direct comparison with the FCE simulations through a representative study of a ten-walled MWCNT. We emphasize that the FCE method provides accurate solution to the deformation morphology and energetics comparable to the fully atomistic simulations. We found that at a relative small twisting angle, the rippling amplitudes $B_{1}(i)$ are nearly zero for all the layers. Thus, the MWCNT does not ripple at this loading regime and the cross sections of all the layers remain circular (see insets in Fig. 3). Upon reaching the bifurcation point, the rippling amplitude increases sharply and the cross sections of 


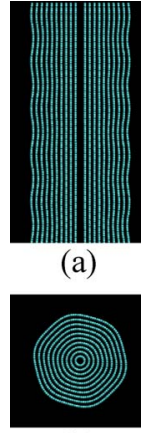

(b)

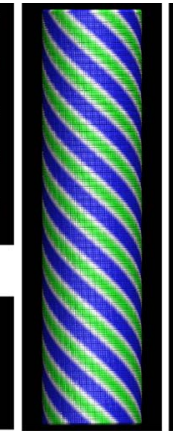

(c)

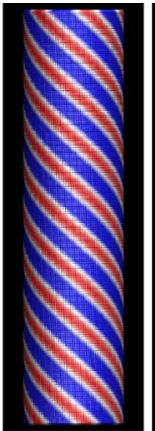

(d)

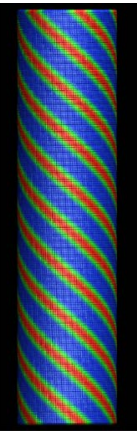

(e)
FIG. 4. (Color online) Rippling deformation of a ten-walled CNT $(5,5) @ \ldots . .(50,50)$ under torsion with $34 \mathrm{~nm}$ in length and $3.4 \mathrm{~nm}$ in radius. (a) Longitudinal view. (b) Cross-sectional view. (c) Deformation map (green for ridges and blue for furrows). (d) Gaussian curvature map (white for zero, blue for negative, and red for positive Gaussian curvature). (e) Energy density map (red for higher energy state and blue for lower).

the outer layers deform from the circular shape first into an oval shape and then into a hexagonal shape with rounded corners. Our coarse-grained simulations predict that the torsional deformation bifurcates at $\phi_{C R}=0.0122 \mathrm{~nm}^{-1}$ with the circumferential wave number $N_{y}=6$. The corresponding evolution of rippling amplitude $B_{1}(i)$ of the $i$ th layer as a function of torsional angle per unit length $\phi$ is shown in Fig. 3. Both the predicted deformation morphology and the wave number agree very well with the FCE model shown in Figs. 2(a) and 2(c). However, the torsion of bifurcation predicted by our model is larger than that of the FCE model $\left(0.0087 \mathrm{~nm}^{-1}\right)$. This is due to the over-constrained shape function adopted by the present coarse-grained model, as compared to the shape functions in finite element based FCE simulations, therefore making the MWCNT artificially more rigid.

Our simulations reveal that the rippling amplitude $B_{1}$ increases monotonically from the inner to the outer layers. Both $B_{1}(1)$ and $B_{1}(2)$ (rippling amplitudes for the innermost two layers) remain nearly zero during the entire loading process. Therefore, the innermost two layers never buckle (always remained as the cylindrical shape) and act as a hard core since developing ripping morphology in these two layers would involve significant strain energy penalty. Such deformation characteristics of the inner two layers have also been found in the FCE simulations. ${ }^{22}$ If the innermost two layers are removed, MWCNTs under torsion develop a local kink rather than a global uniform rippling morphology, ${ }^{30,31}$ a feature commonly seen in twisted SWCNTs.

The simple shape function in Eq. (1) results in a perfect periodic and helical morphology [Figs. 4(a) and 4(b)] at equilibrium state, as shown in Fig. 4, which closely resembles the rippling morphology obtained from the FCE simulations $^{14,22}$ [Figs. 2(a) and 2(c)]. The ridges [outward rippling colored by green in Fig. 4(c)] are identified with positive Gaussian curvatures [red in Fig. 4(d)] and higher strain energy states [red in Fig. 4(e)], while the furrows [inward rippling colored by blue in Fig. 4(c)] are identified with negative Gaussian curvatures [blue in Fig. 4(d)] and lower energy states [blue in Fig. 4(e)]. All these characteristics closely replicate the results of the FCE calculations. Despite (a)

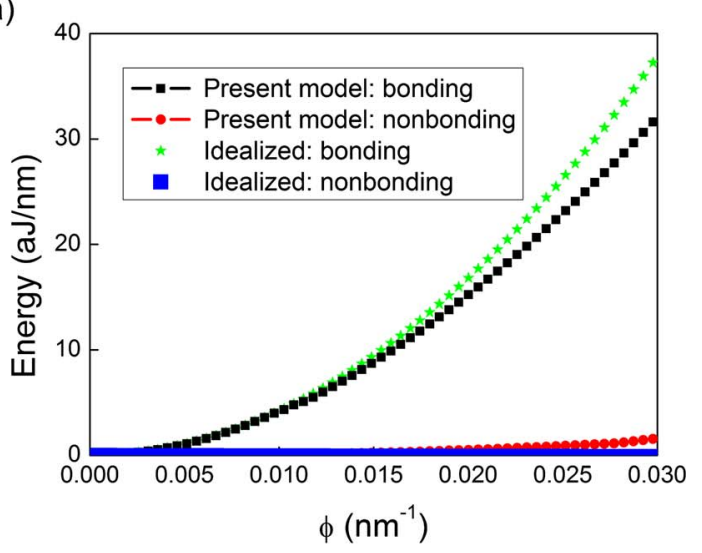

(b)

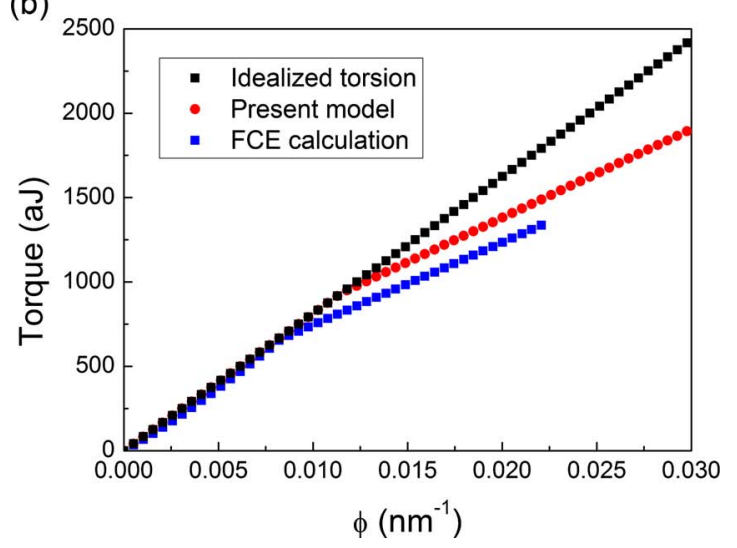

FIG. 5. (Color online) Energetics and mechanical responses of a ten-walled MWCNT under torsion calculated by the present model. For comparisons, an idealized case is also depicted, where the deformation of the MWCNT is constrained to the perfect cylindrical shape without rippling (by fixing $B_{1}$ $=0$ for all the layers) throughout the entire loading process. (a) As compared with the idealized deformation mode, the rippling deformation (actual) beyond the bifurcation point releases the in-plane strain energy, penalized by the increase in the interlayer van der Waals energy. The undeformed configuration is taken as the reference energy state. In the idealized case, the interlayer van der Waals energy is nearly constant throughout the entire loading process, and thus the change in nonbonding energy (blue curve) almost coincides with the horizontal axis. (b) Applied torque as a function of the torsional deformation $\phi$ (torsional angle per unit length). The rippling deformation regime corresponds to a lower $\left(5.514 \times 10^{4} \mathrm{aJ} \mathrm{nm}\right)$ but nearly constant torsional rigidity than the idealized deformation mode $(8.178$ $\times 10^{4} \mathrm{aJ} \mathrm{nm}$ ). The torsion of bifurcation predicted by the present model $\left(1.22 \times 10^{-2} \mathrm{~nm}^{-1}\right)$ is larger than that of the FCE model $(0.87$ $\times 10^{-2} \mathrm{~nm}^{-1}$ ). The post-buckling torsional rigidity predicted by the present model is also slightly higher than that of the FCE calculation (4.891 $\times 10^{4} \mathrm{aJ} \mathrm{nm}$ ), owing to the over-constrained sinusoidal shape function.

the nearly zero rippling amplitude, the innermost layer $(5,5)$ stores a very high strain energy. Owing to the strong confinement, the strain energy in this layer cannot be released via rippling, which may lead to bond breaking and subsequent brittle facture when the torsional deformation continues to increase. $^{17,22}$

It is worth noting that the characteristics of the helical rippling morphology in twisted MWCNTs are different from that in bent MWCNTs, i.e., the so-called Yoshimura or diamond buckling pattern. Structurally, torsion induced rippling is distributed more periodically and uniformly along the tube while bending induced rippling is located only in the compressive side. ${ }^{14,15}$ Energetically, in twisted MWCNTs the ridges stay in a higher energy state than the furrows, while in 
TABLE I. Structural and mechanical properties of MWCNTs with different number of layers. $n$ is the number of layers in a MWCNT. $R$ is tube radius. $\phi_{C R}$ is the torsion of bifurcation. $D_{1}$ and $D_{2}$ are torsional rigidities in the pre- and post-buckling regimes, respectively. Note that $D_{2} / D_{1}$ is nearly constant.

\begin{tabular}{cccccc}
\hline \hline & $\begin{array}{c}R \\
(\mathrm{~nm})\end{array}$ & $\begin{array}{c}\phi_{C R} \\
\left(\mathrm{~nm}^{-1}\right)\end{array}$ & $\begin{array}{c}D_{1} \\
(\mathrm{aJ} \mathrm{nm})\end{array}$ & $\begin{array}{c}D_{2} \\
(\mathrm{aJ} \mathrm{nm})\end{array}$ & $D_{2} / D_{1}$ \\
\hline 10 & 3.40 & $1.22 \times 10^{-2}$ & $8.178 \times 10^{4}$ & $5.514 \times 10^{4}$ & 0.674 \\
20 & 6.80 & $3.83 \times 10^{-3}$ & $1.171 \times 10^{6}$ & $8.017 \times 10^{5}$ & 0.684 \\
30 & 10.20 & $1.92 \times 10^{-3}$ & $5.725 \times 10^{6}$ & $3.959 \times 10^{6}$ & 0.691 \\
40 & 13.60 & $1.04 \times 10^{-3}$ & $1.769 \times 10^{7}$ & $1.253 \times 10^{7}$ & 0.708 \\
50 & 17.00 & $8.72 \times 10^{-4}$ & $4.265 \times 10^{7}$ & $2.888 \times 10^{7}$ & 0.677 \\
60 & 20.40 & $5.23 \times 10^{-4}$ & $8.802 \times 10^{7}$ & $5.779 \times 10^{7}$ & 0.657 \\
70 & 23.80 & $4.19 \times 10^{-4}$ & $1.619 \times 10^{8}$ & $1.098 \times 10^{8}$ & 0.678 \\
80 & 27.20 & $3.49 \times 10^{-4}$ & $2.755 \times 10^{8}$ & $1.867 \times 10^{8}$ & 0.678 \\
90 & 30.60 & $2.79 \times 10^{-4}$ & $4.391 \times 10^{8}$ & $3.034 \times 10^{8}$ & 0.691 \\
100 & 34.00 & $2.44 \times 10^{-4}$ & $6.675 \times 10^{8}$ & $4.467 \times 10^{8}$ & 0.669 \\
\hline \hline
\end{tabular}

bent MWCNTs the strain energy is nearly equally concentrated at the folds (both ridges and furrows). ${ }^{14,15}$

To reveal the physical mechanisms governing the torsion induced rippling deformation, we plot in Fig. 5(a) the evolution of the in-plane strain energy (black curve) and the interlayer nonbonding energy (red curve) as a function of the torsion deformation $\phi$, where the undeformed configuration $(\phi=0)$ is taken to be the reference energy state. For comparisons, an idealized case is also plotted (green and blue curves, respectively), where the deformation of the MWCNT is constrained to the perfect cylindrical shape without rippling (by fixing $B_{1}=0$ for all the layers) throughout the entire loading process. The two sets of curves are graphically indistinguishable up to the bifurcation point and thereafter deviate gradually from each other. In the pre-buckling regime, the in-plane strain energy increases quadratically with respect to $\phi$ and the interlayer nonbonding energy remains almost a constant. Consequently, the mechanical response in this regime can be well characterized within the framework of linear elasticity. ${ }^{5}$ In the post-buckling regime, the interlayer nonbonding energy stored in the rippled morphology is slightly higher compared to the idealized case (the interlayer nonbonding energy for the idealized case hardly changes), while both in-plane strain energy and the total system potential energy are lower. This clearly shows that the in-plane strain energy release is the driving force for rippling, penalized by the increase in interlayer nonbonding energy. Since the in-plane stiffness in MWCNTs is much higher than the out-of-plane stiffness, the rippling deformation represents an effective configurational transition to release the in-plane strain energy, which leads to an energetically favorable state at sufficiently high torsion.

Figure 5(b) shows the torque-torsion relation, where the torque is numerically calculated by Torque $=d E / d \phi$. For the idealized case, the torque linearly increases with torsion throughout the entire loading process (black curve). For the actual case (red curve) where the rippling morphology develops, the torque increases linearly with respect to the torsional deformation up to the buckling point with a torsional rigidity of $D_{1}=8.178 \times 10^{4}$ aJ nm $\left(1 \mathrm{aJ}=10^{-18} \mathrm{~J}\right)$. Despite the nonuniform strain-energy distribution beyond the buckling point [Fig. 4(e)], the overall torque-torsion relation remains almost linear with a reduced torsional rigidity of $D_{2}=5.514$ $\times 10^{4}$ aJ nm, which is slightly higher than the FCE results [blue curve in Fig. $5(\mathrm{~b}), D_{2}=4.89 \times 10^{4} \mathrm{aJ} \mathrm{nm}$ ]. In this figure, the critical twisting angle at which rippling morphology initiates can be more easily identified from the torque-torsion relation. It should be noted that according to the results in Ref. 15 , the torque-torsion relation is not strictly bilinear, as it is argued that the strain energy-torsion relation for thick MWCNTs follows

$$
E / L=C\left\{\begin{array}{ccc}
(\gamma R)^{2} & \text { for } & |\gamma R| \leq \ell_{c r} \\
\ell_{c r}^{2-a}|\gamma R|^{a} & \text { for } & |\gamma R|>\ell_{c r}
\end{array},\right.
$$

where $C$ is a material constant, $R$ is the outer radius, $\gamma$ $=R \phi$ is the torsional strain, $\ell_{c r}$ is a critical length scale which is a constant around $0.1 \mathrm{~nm}$, and $a$ is an anharmonic exponent close to 1.6. According to this law, in the pre-buckling regime the torque-torsion relation is indeed linear, but after bifurcation, the torque is proportional to $\phi^{a-1}$. However, for moderate (reasonable) torsional strains, given the parameter values, the post-buckling response very closely follows a linear law. In bending, ${ }^{14,15}$ the anharmonic exponent is smaller, and the post-buckling effective modulus depends more strongly on deformation.

\section{B. Size dependence and scaling law}

The representative study on the ten-walled MWCNT shows that both the deformation evolution and the energetics predicted by the present model agree reasonably well with the FCE results, thus validating the coarse-grained method. This agreement is obtained with a drastically reduced computational cost. Indeed, for the ten-walled MWCNT studied, the fully atomistic model consists of more than 450 $\times 10^{3}$ degrees of freedom; a typical FCE model consists of about $54 \times 10^{3}$ degrees of freedom, while the present model has only 22 independent variables. To explore the sizedependent mechanical response of twisted MWCNTs and to further demonstrate the efficiency of the present coarsegrained model, we now analyze the deformation energetics and morphologies of a series of MWCNTs with up to 100 layers. Note that the thick MWCNTs considered here are computationally very costly for the FCE model and unafford- 
able for fully atomistic simulations. The structural and mechanical properties for these MWCNTs are summarized in Table I.

If one models the $n$-walled MWCNTs as a set of concentrically arranged elastic thin shells by linear elasticity without taking into account the interlayer coupling, the torsional rigidity of the MWCNT is ought to scale with $n^{2}$ $\times(n+1)^{2}$. In the light of this classical scaling law, we scale the pre- and post-buckling torsional rigidities $D_{1}$ and $D_{2}$ for all the tubes by $n^{2} \times(n+1)^{2}$, denoted by $\bar{D}_{1}$ and $\bar{D}_{2}$, respectively, and plot them as a function of $n$, as shown in Fig. 6(a). We find that both pre- and post-buckling torsional rigidities of all the MWCNTs considered follow the simple scaling law, i.e., the scaled values are almost constant. The postbuckling rigidity is found to be slightly lower than the prebuckling rigidity. The ratio $D_{2} / D_{1}$ (also $\bar{D}_{2} / \bar{D}_{1}$ ) is nearly a constant of $0.681 \pm 0.012$, which is slightly higher than that obtained from the FCE calculations $[0.638 \pm 0.013$ (Fig. 6(b))]. Again, we attribute this discrepancy to the overconstrained shape function adopted in our coarse-grained method. The magnitude of the discrepancy is relatively small, particularly considering the drastic reduction in complexity of the model. While the linear elastic response in the pre-buckling regime is expected and mainly governed by the in-plane deformation energetics, we conjecture that the scaling law in the post-buckling regime is a result of compatible deformation of all the layers as a competition of in-plane and interlayer interactions. Note that in the limit of super-thick MWCNTs this scaling law can be approximated by $n^{4}$, while in the limit of thin MWCNTs (less than ten layers) the scaling law no longer holds with more than $\sim 20 \%$ difference.

Going back to the law in Eq. (2) proposed in Ref. 15, taking two derivatives with respect to the torsional strain $\gamma$ $=R \phi$ we compute the elastic modulus. According to this relation, the post-buckling modulus $D_{2}$ does depend on deformation, but only slightly. Evaluating this modulus at the critical strain, it follows from a simple calculation that $D_{2} / D_{1}=a(a-1) / 2$, in agreement with the above observation that this ratio is independent of the tube radius. Additionally, this observation shows that the anharmonic exponent can be computed from the ratio of the pre- and post-buckling stiffness, which can in principle be measured experimentally.

Following the similar analysis based on the elastic thin shell theory, the stability of an individual shell decreases when its radius increases, and the outermost layer in a MWCNT is least stable. Under torsion, the shear strain applied to each layer scales with $R$. Taken together, one reaches that the buckling point $\phi_{C R}$ of a MWCNT may scale with $R^{-2}{ }^{15}$ This suggests that a thicker MWCNT ripples at a smaller torsion. Indeed, our model predicts that the torsion of bifurcation of the 100-walled CNT is only 2.44 $\times 10^{-4} \mathrm{~nm}^{-1}$, about two orders of magnitude smaller than that of the ten-walled CNT. However, careful curving fitting of our simulation data for all the MWCNTs studied reveals that the torsion of bifurcation $\phi_{C R}$ scales with $R^{-1.72}$, inconsistent with the classical elasticity theory. This scaling law is compared to $R^{-1.82}$ obtained from FCE simulations. ${ }^{31}$ Again, the over-constrained shape function in the present model ac- (a)

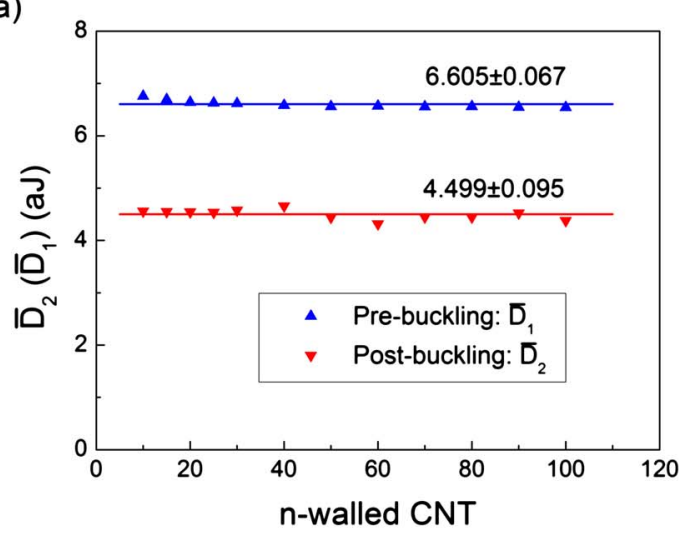

(b)

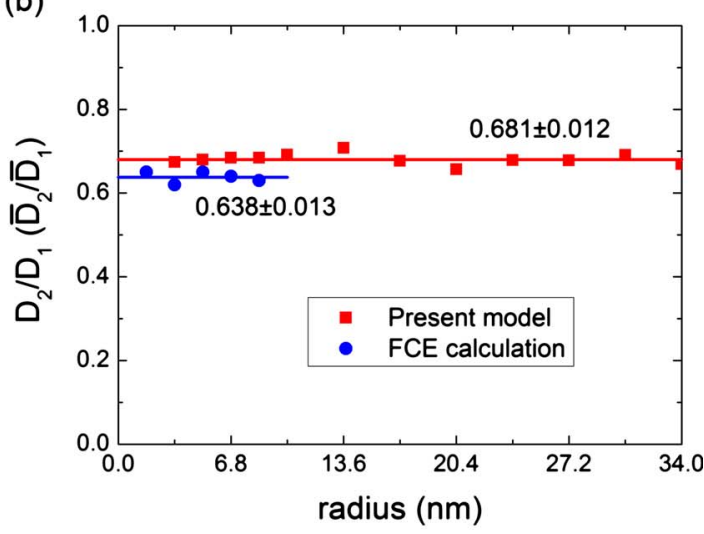

(c)

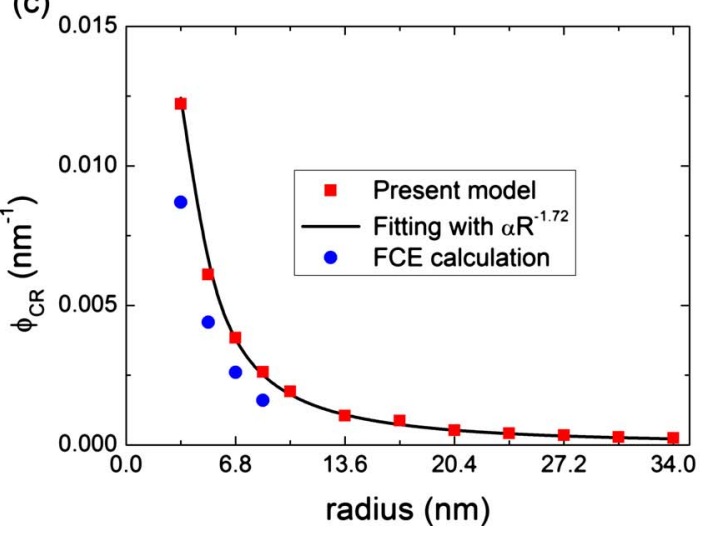

FIG. 6. (Color online) The scaling law of the twisted MWCNTs with up to 100 layers. Results from the FCE simulations are also presented for comparisons. (a) The scaled torsional rigidities in the pre- and post-buckling regimes as a function of number of layers $n$ in a MWCNT, respectively. Both the scaled torsional rigidities are nearly constants. (b) The ratio between the torsional rigidities in the pre- and post-buckling regimes is nearly a constant of $0.681 \pm 0.012$. Results from the FCE model reveal that the ratio is $0.638 \pm 0.013$. (c) The torsion of bifurcation $\phi_{C R}$ scales with $R^{-1.72}$.

counts for the discrepancy of the scaling exponents between the present model and the FCE model. Here, the effect of the simple shape function used may be more drastic, since even though the post-buckling regime of twisted MWCNTs is very well characterized by the sinusoidal shape functions, the onset of buckling does, in general, mobilize different deformation modes. ${ }^{16}$ As a natural extrapolation of our simulation results, super-thick MWCNTs would ripple at a vanishing critical torsion. In this limit, the MWCNTs behave as bulk beams, and the mechanical response of the MWCNTs can be fully characterized by the post-buckling rigidity. 


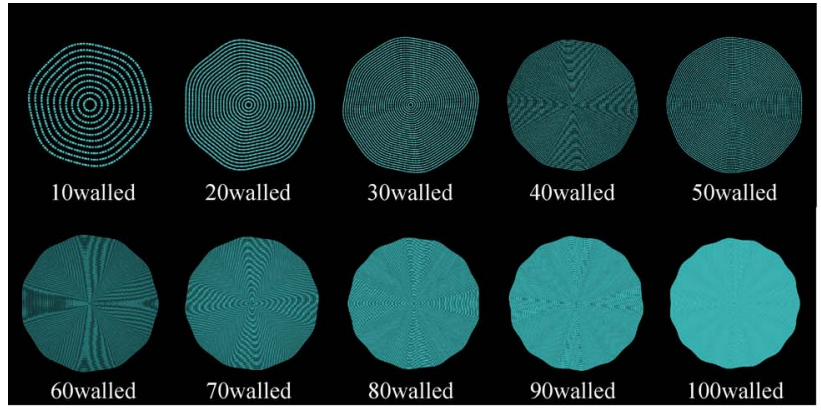

(a)

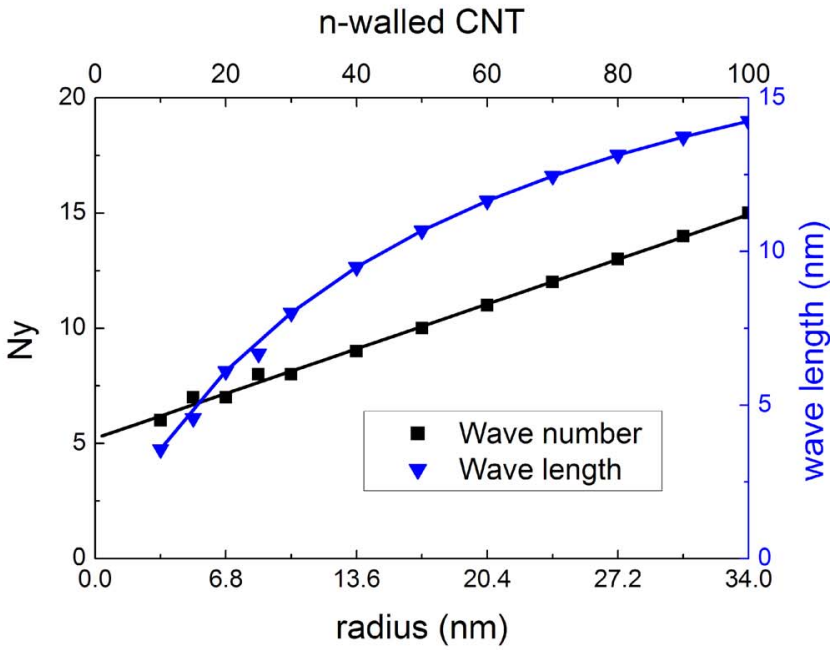

(b)

FIG. 7. (Color online) Rippling morphology of $n$-walled CNTs under torsion with $n=10,20, \ldots, 100$, respectively. (a) The cross-sectional deformation morphologies. (b) The circumferential wave number $N_{y}$ increases nearly linearly with the number of layers $n$ and tube radius $R$. Correspondingly, the wavelength in circumferential direction monotonically increases with an upper limit of $\sim 21.3 \mathrm{~nm}$ as the tube radius approaches infinity.

The examination of the cross-sectional morphology of the MWCNTs shows that the initial circular shape turns into a polygonal shape with rounded corners [Fig. 7(a)]. We find that the wave number $N_{y}$ in the circumferential direction increases nearly linearly with the number of layers $n$, as shown in Fig. 7(b) (black curve). By curve fitting, one finds that the linear relationship can be described by $N_{y}=\operatorname{Int}(0.1 n+4)$, where the symbol Int denotes taking the integer of the value in the parenthesis. Since the circumference of an $n$-layered MWCNT is about $c=2.13 n$ (considering all the layers as armchair tubes), the circumferential wavelength $\lambda$, defined as the circumference divided by the circumferential wave number $N_{y}$, increases monotonically with $n$ and $R$ [blue curve in Fig. 7(b)]. In the limit of super-thick MWCNTs $(R \rightarrow \infty)$, one finds the upper limit of the wavelength in the circumferential direction of $\sim 21.3 \mathrm{~nm}$.

\section{DISCUSSION AND CONCLUSIONS}

In summary, the present study develops a coarse-grained scheme to characterize the deformation energetics and morphology of twisted MWCNTs. The method involves approximating the periodic wave-like rippling morphology by a simple sinusoidal shape function with only a few parameters, which are determined by the geometry optimization. The method is carefully validated through a representative study with comparisons to the FCE simulations. This method shows clear advantages over MD and FCE simulations in that it reduces the computational complexity by several orders of magnitude while still captures the essential deformation characteristics and mechanical properties of twisted MWCNTs. With appropriately chosen shape functions, the method can be easily extended to the bent MWCNTs.

The high efficiency of the present coarse-grained method enables systematic studies of the deformation energetics and morphology of super-thick MWCNTs. Our coarse-grained simulations reveal that the rippling morphology is a result of in-plane strain energy release, penalized by the increase in the interlayer van der Waals energy. Due to the resisting role of the interlayer van der Waals interactions to the rippling deformation, the simplified treatment of MWCNTs as noninteracting elastic thin shells predicts a smaller buckling point and overestimates the rippling amplitude.

Although the rippling deformation pattern is intrinsically nonlinear, our simulation results show that the mechanical response can be well characterized by a simple bilinear law for practical deformation ranges. The ratio between the torsional rigidities in the pre- and post-buckling regimes is nearly a constant of about 0.681 , independent of the tube radius. We also find that the bifurcation torsional strain powerly scales with the tube radius. The bilinear constitutive relation and the scaling law of the bifurcation torsional strain constitute a simple nonlinear beam theory, which may be further incorporated into finite element analysis for large scale simulations of CNT-based materials and devices.

Despite its great advantages in computational simplicity and significantly enhanced computational affordability, the use of the over-constrained shape function in the present method makes the MWCNTs artificially more rigid, giving rise to slightly higher bifurcation torsional strains and torsional rigidities. Such an artifact can be minimized by adding high frequency terms in the shape function; this, however, increases the computational complexity. In addition, one should note that the present method simulates a segment of uniformly twisted MWCNT without imposing any additional boundary conditions to the tube ends. This naturally eliminates the boundary effects, which were proven to introduce inaccuracies in simulations of short MWCNTs. ${ }^{15}$

It is worth pointing out that the scaling law developed in this study only applies to MWCNTs with at least ten layers. Large discrepancies appear for MWCNTs with less than ten layers. In the limit of a SWCNT, local kinks develop as the characteristic deformation morphology beyond the buckling point, rather than the global rippling morphology in twisted thick MWCNTs. On the other side, super-thick tubes can be considered as a bulk beam, which are well characterized by the classical beam theory. In this limiting case, the MWCNTs buckle instantaneously upon a vanishing twist and only the post-buckling regime is operative.

\section{ACKNOWLEDGMENTS}

We gratefully acknowledge the grant support from the National Science Foundation grant under Awards No. 
0600661 (0826841) and 0600642 (Clark V. Cooper, program manager). M. A. acknowledges the support of the European Commission (MIRG-CT-2005-029178) and the Ministerio de Ciencia e Innovacion (DPI2007-61054).

${ }^{1}$ R. H. Baughman, A. A. Zakhidov, and W. A. de Heer, Science 297, 787 (2002).

${ }^{2}$ H. Rafii-Tabar, Phys. Rep. 390, 235 (2004).

${ }^{3}$ R. Khare, S. L. Mielke, J. T. Paci, S. L. Zhang, R. Ballarini, G. C. Schatz, and T. Belytschko, Phys. Rev. B 75, 075412 (2007).

${ }^{4}$ S. L. Zhang, T. Zhu, and T. Belytschko, Phys. Rev. B 76, 094114 (2007).

${ }^{5}$ L. F. Wang, Q. S. Zheng, J. Z. Liu, and Q. Jiang, Phys. Rev. Lett. 95 105501 (2005)

${ }^{6}$ S. L. Zhang, S. L. Mielke, R. Khare, D. Troya, R. S. Ruoff, G. C. Schatz, and T. Belytschko, Phys. Rev. B 71, 115403 (2005).

${ }^{7}$ T. Dumitrica, M. Hua, and B. I. Yakobson, Proc. Natl. Acad. Sci. U.S.A. 103, 6105 (2006)

${ }^{8}$ S. L. Zhang, R. Khare, T. Belytschko, K. J. Hsia, S. L. Mielke, and G. C. Schatz, Phys. Rev. B 73, 075423 (2006).

${ }^{9}$ S. L. Mielke, S. L. Zhang, R. Khare, R. S. Ruoff, T. Belytschko, and G. C. Schatz, Chem. Phys. Lett. 446, 128 (2007).

${ }^{10}$ S. L. Zhang, R. Khare, Q. Lu, and T. Belytschko, Int. J. Numer. Methods Eng. 70, 913 (2007).

${ }^{11}$ A. Pantano, D. M. Parks, and M. C. Boyce, J. Mech. Phys. Solids 52, 789 (2004).

${ }^{12}$ M. Arroyo and T. Belytschko, J. Mech. Phys. Solids 50, 1941 (2002).

${ }^{13}$ M. Arroyo and T. Belytschko, Phys. Rev. B 69, 115415 (2004).

${ }^{14}$ M. Arroyo and T. Belytschko, Phys. Rev. Lett. 91, 215505 (2003).

${ }^{15}$ I. Arias and M. Arroyo, Phys. Rev. Lett. 100, 085503 (2008).
${ }^{16}$ M. Arroyo and I. Arias, J. Mech. Phys. Solids 56, 1224 (2008).

${ }^{17}$ X. Y. Li, W. Yang, and B. Liu, Phys. Rev. Lett. 98, 205502 (2007).

${ }^{18}$ M. F. Yu, O. Lourie, M. J. Dyer, K. Moloni, T. F. Kelly, and R. S. Ruoff, Science 287, 637 (2000).

${ }^{19}$ J. Y. Huang, S. Chen, Z. Q. Wang, K. Kempa, Y. M. Wang, S. H. Jo, G. Chen, M. S. Dresselhaus, and Z. F. Ren, Nature (London) 439, 281 (2006).

${ }^{20}$ P. Zhang, Y. Huang, P. H. Geubelle, P. A. Klein, and K. C. Hwang, Int. J. Solids Struct. 39, 3893 (2002).

${ }^{21}$ The FCE method involves coarse graining both the in-plane covalent binding energy and the interlayer van der Waals interaction energy. To avoid the nonphysical interpenetrating between the neighboring nanotube layers, at least 12 quadrature points are needed for each finite element in approximating the interlayer interaction energy, making the computation very expensive.

${ }^{22}$ X. Huang, J. Zou, and S. L. Zhang, Appl. Phys. Lett. 93, 031915 (2008).

${ }^{23}$ Z. H. Xia, P. R. Guduru, and W. A. Curtin, Phys. Rev. Lett. 98, 245501 (2007).

${ }^{24}$ D. Qian, E. C. Dickey, R. Andrews, and T. Rantell, Appl. Phys. Lett. 76, 2868 (2000).

${ }^{25}$ T. V. Sreekumar, T. Liu, B. G. Min, H. Guo, S. Kumar, R. H. Hauge, and R. E. Smalley, Adv. Mater. (Weinheim, Ger.) 16, 58 (2004).

${ }^{26}$ Z. Qin, X. Q. Feng, J. Zou, Y. J. Yin, and S. W. Yu, Appl. Phys. Lett. 91, 043108 (2007).

${ }^{27}$ D. W. Brenner, O. A. Shenderova, J. A. Harrison, S. J. Stuart, B. Ni, and S. B. Sinnott, J. Phys.: Condens. Matter 14, 783 (2002).

${ }^{28}$ L. A. Girifalco, M. Hodak, and R. S. Lee, Phys. Rev. B 62, 13104 (2000).

${ }^{29}$ D. C. Liu and J. Nocedal, Math. Program. 45, 503 (1989).

${ }^{30}$ T. C. Chang and J. Hou, J. Appl. Phys. 100, 114327 (2006).

${ }^{31} \mathrm{X}$. Huang, J. Zou, and S. Zhang (unpublished). 